\title{
Real Time Warning Model of Transmission Tower Tilt Based on Multi-Sensor Data
}

\author{
Xie HU, Huikun $\mathrm{PEI}^{\mathrm{a}}$, Bingcai LIU ${ }^{\mathrm{a}}$, Chen WANG ${ }^{\mathrm{a}}$, Changjin $\mathrm{HAO}^{\mathrm{b} 1}$ \\ ${ }^{a}$ Shenzhen Power Supply Bureau Co, Ltd. China Southern Power Grid, Shen Zhen \\ 518001, Guangdong Province, China \\ ${ }^{b}$ Institute of Energy Sensing and Information, Sichuan Energy Internet Research \\ Institute, Tsinghua University, Chengdu 610000, China
}

\begin{abstract}
Transmission line operation environment is complex, prone to tilt collapse accident, so a transmission tower tilt real-time early warning model based on multi-sensor data is established to judge whether the tower is stable operation by detecting the tilt state of the early warning tower. The pressure and inclination sensors are deployed at different positions of the transmission tower to collect the inclination and stress of the tower in real time, and transmit them to the remote monitoring terminal through the wireless network to send out an alarm. The operators can timely adjust according to the alarm situation to maintain the safe operation of the transmission line. The experimental results show that the model can realize the real-time warning of transmission tower tilt, the measurement accuracy can meet the needs of comprehensive detection of tower state, and the application can effectively ensure the safety of staff and reduce the work intensity.
\end{abstract}

\section{Introduction}

Power grid transmission tower has the characteristics of large span, long span and large flexibility, which leads to the difficulty and long cycle of staff inspection, and the difficulty of operation and maintenance of some key nodes. It is urgent to carry out real-time on-line detection of tower tilt state through effective technical path, find problems in time and give an alarm, so as to maintain the safe operation of transmission lines, reduce the work intensity of inspection, and improve the management efficiency of power grid.

In reference [1], using geographic information system (GIS) technology and taking the impact energy of slope as the disaster intensity, a landslide probability model which can be used to predict the landslide activity time is proposed, reflecting the quantitative functional relationship of the damage degree of transmission towers in landslide prone areas. The proposed method is feasible and reasonable. However, a lot of preparations need to be done before the method is applied, such as the collection and sorting of historical landslide data, the evaporation and loss properties of rainwater in different soils, the information of landslide geological conditions and the structural parameters of transmission tower. In reference [2], a new all-weather sensing technology is introduced to detect the power tower of the whole scene synthetic aperture radar (SAR) image, so as to realize the rapid assessment of large-scale

${ }^{1}$ Corresponding author: Changjin Hao, Institute of Energy Sensing and Information, Sichuan Energy Internet Research Institute, Tsinghua University, Chengdu 610000, China; E-mail: 1527860368@qq.com. 
transmission line disaster. However, this method is difficult to implement on site. In reference [3], based on the principle of corrosion electrochemistry, a corrosion monitoring sensor based on "strip groove" structure was proposed, the corrosion mechanism of tower foot was studied, the corrosion monitoring method of tower foot was studied, and a set of data monitoring system was developed. However, this method can only monitor tower faults caused by corrosion. In reference [4], the online monitoring technology of tower foundation deformation of transmission lines is developed based on fiber Bragg grating stress sensor. But the cost of field application of this method is relatively high. According to the previous studies, most of the previous studies are based on a single parameter and cannot fully monitor the state of the tower.

In this paper, a real-time warning model of transmission tower tilt based on multisensor data is proposed. The tower tilt data is detected by multiple pressure and tilt sensors. After data fusion processing, it is reflected to the operation and maintenance staff of remote monitoring terminal through wireless communication to remind the staff of tower tilt in real time and effectively, Real time detection and early warning of transmission tower tilt state are realized.

\section{Real Time Warning Model of Transmission Tower Tilt Based on Multi-Sensor Data}

\subsection{Overall structure of real time warning model for transmission tower inclination}

Based on the multi-sensor data, a real-time early warning model of transmission tower tilt is established, which is divided into three control levels: field control, centralized control and remote control. Among them, the equipment used to detect the tilt and stress state of transmission tower in real time, such as tension sensor, tilt sensor and detection terminal [5-6], is collectively referred to as the field control layer. By using concentrator, ZigBee technology and GPRS communication module deployed in transmission tower, all tower tilt and tension information are collected from the detection terminal. The equipment of ZigBee and GPRS data frame format conversion is called the centralized control layer. The monitoring terminal with the functions of digital processing and data storage of concentrator tower deployed in the remote monitoring center is called remote control layer. The staff can adjust according to the digital measurement value [7-8]. The structure of the model is shown in Figure 1.

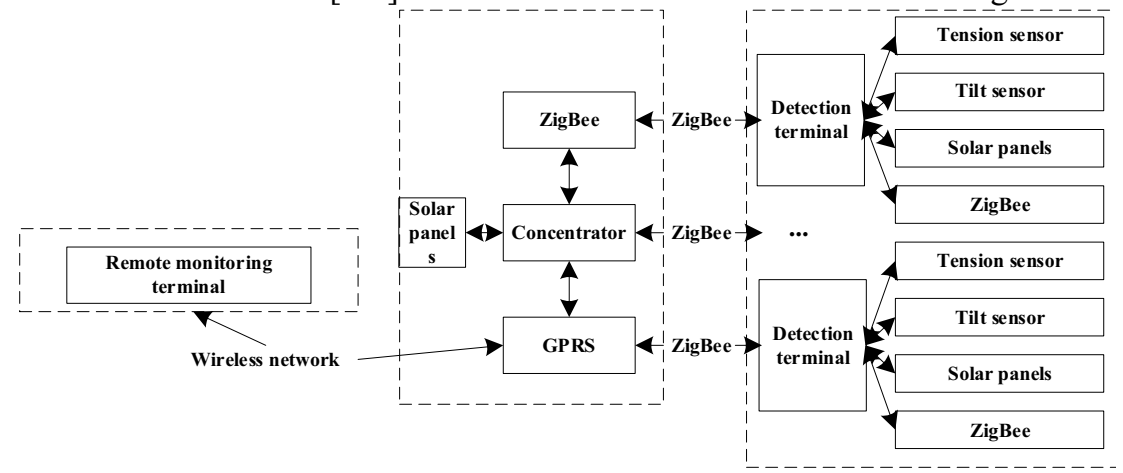

Figure 1. Real time warning structure diagram of transmission tower inclination. 


\subsection{Multi Sensor Data Fusion Processing}

The stability of transmission tower is judged by fusing the data collected by multisensor. The treatment process is as follows:

Step1: The sensor data is filtered by discrete wavelet transform, and the continuous wavelet transform of the data $x(t)$ collected by each sensor is defined as:

$$
W x(j, n)=\int_{-\infty}^{\infty} x(t) \psi_{j, n}^{*}(t) d t
$$

The wavelet basis function is as follows:

$$
\psi_{j, n}(t)=2^{-j / 2} \psi\left(2^{-j} t-n\right)
$$

Where, $j$ and $n$ are scale and translation factors respectively. The limitation of wavelet basis function and Fourier transform can be ensured by selecting appropriate mother wavelet $\varphi(t)$, so wavelet analysis adopts time-frequency analysis.

In the process of discrete wavelet transform, it is necessary to discretize the scale factor according to the power series to realize the uniform discretization of the translation factor [9]. The discrete wavelet transform formula of the data collected by each sensor is obtained as follows:

$$
W x(j, n)=\int_{-\infty}^{\infty} x(t) \psi_{j, n}^{*}(t) d t
$$

Step2: Based on the wavelet packet energy spectrum strategy, the pressure and inclination data are decomposed, and the feature vectors are extracted:

$$
E_{j}=\sum_{k=1}^{N}\left|x_{j k}\right|^{2}
$$

Where $E_{j}$ is the frequency band component of the $j$ th sensor and $X_{j k}$ is the value of each discrete point, $k=1,2,3 \ldots, N$. The proportion formula of each frequency band energy in the total energy is calculated as follows:

$$
e_{i}=\frac{E_{i}}{\sum E_{n}}
$$

Where $E_{n}$ is the frequency band component of the $n$th sensor and represents the input characteristic quantity of stability diagnosis.

Step 3: RBF neural network is used to diagnose the stability of transmission tower. RBF neural network mainly includes input-output and hidden three layers, which can realize nonlinear mapping. 


$$
\begin{gathered}
f_{n}(e)=\omega_{0}+\sum_{i=1}^{n} \omega_{i} \phi\left(\left\|e-c_{i}\right\|\right) \\
\phi\left(\left\|e-c_{i}\right\|\right)=\exp \left(\frac{\left\|e-c_{i}\right\|^{2}}{\sigma_{i}^{2}}\right)
\end{gathered}
$$

Among them, $e$ and $\omega_{j}$ represent the input vector and weight respectively, $c_{j}$ represents the data center of the basis function, $\sigma_{j}$ and $n$ represent its width and number respectively, $\varphi$ and $\omega_{0}$ represent the radial basis function and deviation respectively.

Step 4: The results are fused by Bayesian reasoning to obtain the final results as follows:

$$
\begin{gathered}
u(k)=\left\{\begin{array}{l} 
\pm 1, a_{0}(k)>0 \\
-1, a_{0}(k) \leq 0
\end{array}\right. \\
a_{0}(k)=\log \frac{P_{k}\left(H_{1} / u_{1}(k), \mathrm{L}, u_{i}(k), \mathrm{L} u_{n}(k)\right)}{P_{k}\left(H_{0} / u_{1}(k), \mathrm{L}, u_{i}(k), \mathrm{L} u_{n}(k)\right)}
\end{gathered}
$$

Where $u(k)$ and $u_{\mathrm{n}}(k)$ are binary functions, the posterior probability of $H_{i}$ after the k-th measurement is described by $P_{k}\left(H_{i} / u_{1}(k), \mathrm{L}, u_{i}(k), \mathrm{L} u_{n}(k)\right), i=0.1$, and $H_{0}$ and $H_{1}$ respectively indicate that there is the data in the target.

\subsection{On Line Comprehensive Detection Process of Tower State}

The gravity acceleration on the $\mathrm{X}, \mathrm{y}$ and $\mathrm{Z}$ axes collected by the sensor is taken as the measurement target to obtain the inclination of the tower in all directions. Firstly, the acceleration of the tower needs to be changed into the inclination. The horizontal direction, the direction perpendicular to the horizontal direction and the direction perpendicular to the horizontal plane are defined as $\mathrm{X}, \mathrm{Y}$ and $\mathrm{Z}$ axes respectively. Based on these axes, a space rectangular coordinate system is constructed to make the included angles of the positive direction of $\mathrm{X}$, Yand $\mathrm{Z}$ axes in the case of tower tilt as follows $\alpha, \beta, \gamma$. Figure 2 is the software flow chart of tower inclination detection device.

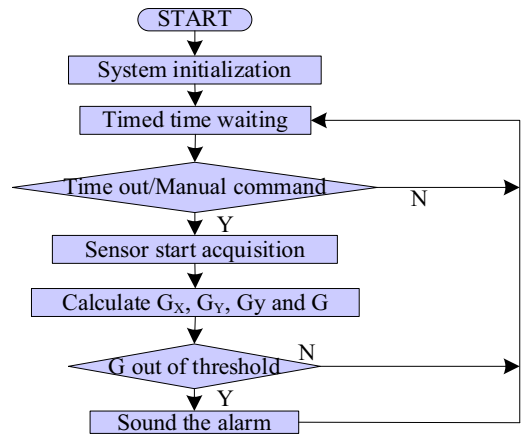

Figure 2. Treatment process of tower inclination monitoring device. 
In order to reduce the power loss of transmission tower, the timing data acquisition is selected. When the set threshold is reached, the sensor transmits the inclination data to the equipment control unit and calculates the inclination to determine whether the comprehensive inclination exceeds the threshold and whether the alarm is executed.

The angle between the tower and the positive direction of $\mathrm{X}, \mathrm{Y}$ and $\mathrm{Z}$ axes when the tower is inclined $\alpha, \beta, \gamma$. The calculation formulas are as follows:

$$
\begin{aligned}
& \alpha=\arctan \left(\frac{G_{X}}{\sqrt{G_{Y}^{2}+G_{Z}^{2}}}\right) \\
& \beta=\arctan \left(\frac{G_{Y}}{\sqrt{G_{X}^{2}+G_{Z}^{2}}}\right) \\
& \gamma=\arctan \left(\frac{\sqrt{G_{X}^{2}+G_{Y}^{2}}}{G_{Z}}\right)
\end{aligned}
$$

The gravity acceleration components on $\mathrm{X}, \mathrm{Y}$ and $\mathrm{Z}$ axes are respectively $G_{X}, G_{Y}$, and $G_{Z}$, the value of this component can be positive or negative. When its value is positive, it means that the direction of gravity acceleration is equal to the positive direction of $\mathrm{X}, \mathrm{y}$ and $\mathrm{Z}$ axes. When its value is negative, it is opposite $\alpha, \beta, \gamma$. The value range of is $\left[-90^{\circ},+90^{\circ}\right]$. The calculation formula of inclination $G$ is as follows:

$$
G=\sqrt{\left(G_{X} \cdot G_{X}+G_{Y} \cdot G_{Y}+G_{Z} \cdot G_{Z}\right)}
$$

According to the comprehensive inclination, the alarm threshold, warning threshold, prompt threshold and normal value of each tower can be set.

\section{Experimental Analysis}

In order to analyze the performance of the model, the test and analysis are carried out. The analysis results of tower tilt data collected by this model are shown in Table.1.

Table 1. Analysis results.

\begin{tabular}{cccccccc}
\hline Serial number & Wind direction & Route direction & $\alpha /^{\circ}$ & $\beta /{ }^{\circ}$ & $\gamma /{ }^{\circ}$ & $G$ & Wind-force \\
\hline 1 & Northwest wind & East West & 76 & 68 & 54 & 1.2 & 8 \\
2 & West wind & North South & 20 & 35 & 68 & 1.1 & 8 \\
3 & Southwest wind & North South & 15 & 45 & 84 & 1.1 & 8 \\
4 & Northeast wind & North South & -89 & -74 & -68 & 1.2 & 8 \\
5 & Southeast wind & East West & -55 & -84 & -46 & 1.8 & 9 \\
6 & East wind & North South & 61 & 75 & 68 & 1.9 & 9 \\
7 & North wind & North South & 48 & 36 & 48 & 0.6 & 7 \\
8 & North wind & North South & 21 & 48 & 15 & 0.8 & 7 \\
9 & North wind & North South & 43 & 81 & 49 & 0.9 & 7 \\
10 & North wind & East West & -48 & -15 & -54 & 1.2 & 7 \\
\hline
\end{tabular}


Through the analysis of Table 1, it can be seen that under the condition of 7-level north wind, the route of line 7 is north-south, and the comprehensive inclination degree is the lowest. Under the condition of 9-level southeast wind, the comprehensive inclination degree of line 5 is the highest.

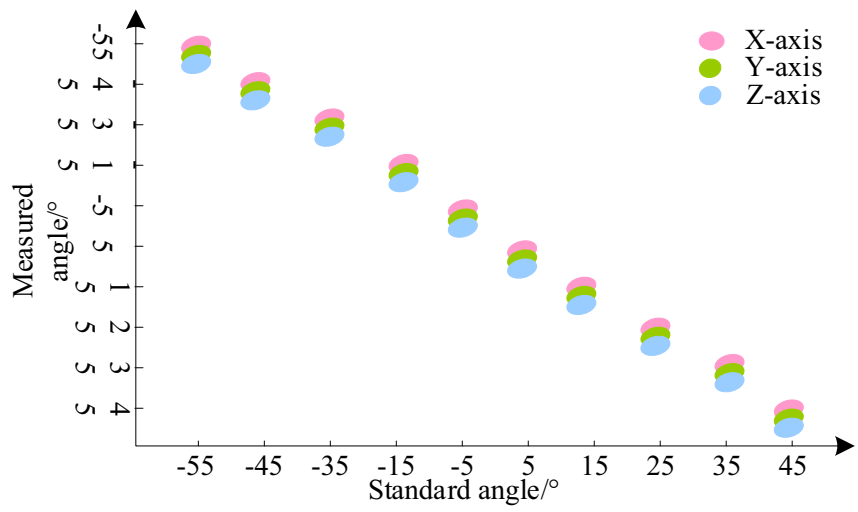

Figure 3. Analysis results of sensor inclination error.

According to the analysis of Figure 3, there are different errors in the angle measured by different axial tower tilt angles, and the average error of $\mathrm{x}$-axis is $0.14^{\circ}$, The maximum error is only $0.19^{\circ}$, The average error of $y$-axis is $0.21^{\circ}$, The maximum error is $0.24^{\circ}$, The average error of $\mathrm{Z}$ axis is $0.19^{\circ}$, The maximum error is $0.23^{\circ}$, Therefore, the measurement accuracy of this model can meet the needs of tower state comprehensive detection.

TE (technical efficiency change index), PTEC (pure technical efficiency change index) and SEC (scale efficiency change index) are used to measure the information utilization rate of the model. When the value of the three indexes is greater than 1 , it means that the model has higher information utilization rate. When TE $>1$, it means that the model has higher technical efficiency, When PREC $>1$, it means that the model has high technology maturity, and when SEC $>1$, it means that the model has good scale effect. Test the information utilization rate of this model within one month, as shown in Figure 4.

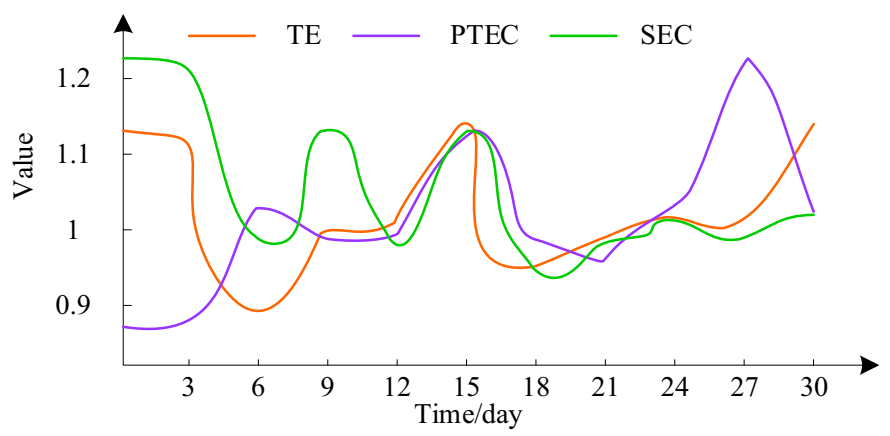

Figure 4. Information utilization test results.

According to the test results in Figure 4, the product of TE, PTEC and SEC of this model is greater than 1 , and the average values are 1.049, 1.049 and 1.059, respectively, all large than 1 , which indicates that this model has higher information utilization rate, 
better technical level and higher scale efficiency, which can enrich the detailed information of sensor data collection and improve the safety early warning effect.

The missing alarm rate and false alarm rate represent the proportion of positive and negative samples in negative and positive samples respectively. According to the statistics, this paper uses the model to detect the missing alarm rate and false alarm rate of different types of indicators of transmission tower tilt real-time warning, and analyzes whether it can achieve the expected goal. The test results are shown in Figure 5 .

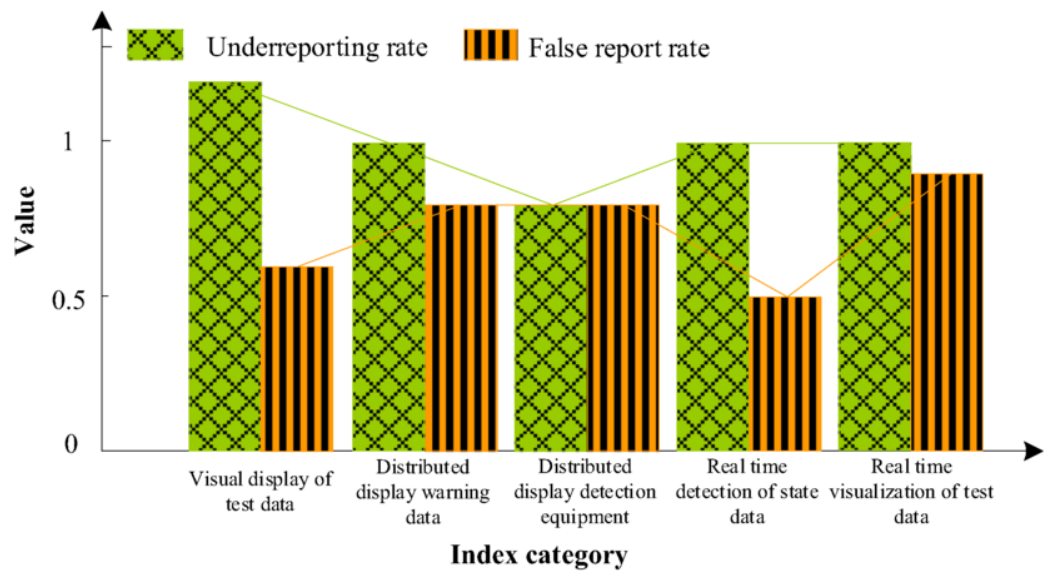

Figure 5. Comparison of missed alarm rate and false alarm rate of different types of indicators.

According to the test results of the model in Figure 5, the leakage rate and false alarm rate of different types of indicators are obviously lower by using this model to detect the tilt of transmission towers, which is not more than $1.5 \%$. It shows that the model can correctly display the specific modules of the real-time warning of transmission tower tilt in practical application. The accuracy of the model detection samples is high and meets the expected target, The tower status can be visualized at the remote monitoring terminal for the staff.

The early warning accuracy performance of this model is analyzed on KDD-99 and CICIDS2017 data sets, and the results are shown in Figure 6.

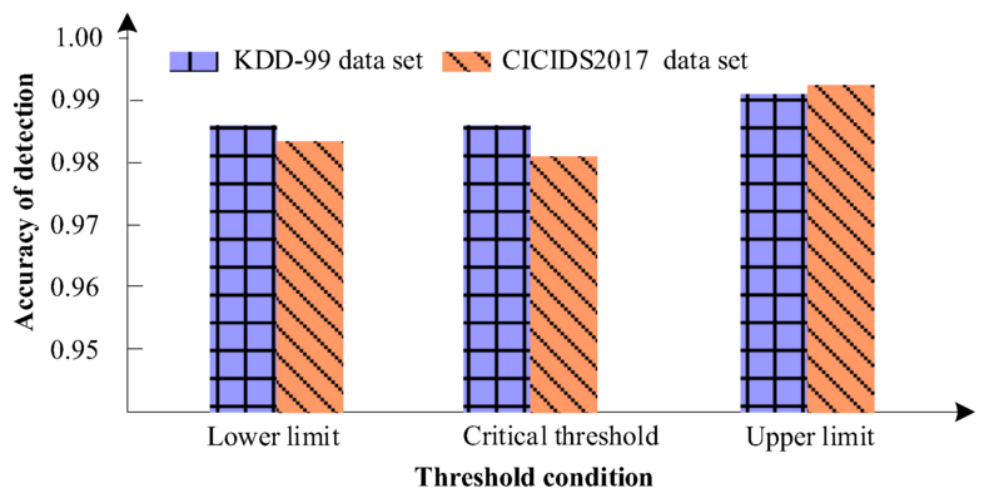

Figure 6. Early warning accuracy under correlation threshold. 
It can be seen from Figure 6 that the model has high detection accuracy under the conditions of lower threshold and critical threshold, both of which are higher than $97 \%$, and the detection accuracy near the upper threshold is higher than $99 \%$. It shows that the real-time warning model of transmission tower tilt based on multi-sensor data has good practical application effect.

The comparison of the actual application effect is shown in Figure 7. It can be seen from Figure 7 that the real-time early warning model of transmission tower inclination based on multi-sensor data proposed in this paper has good practical application effect. By comparing the effects before and after application, it is found that the model can effectively ensure the personal safety of workers, reduce their work intensity and improve the maintenance quality.

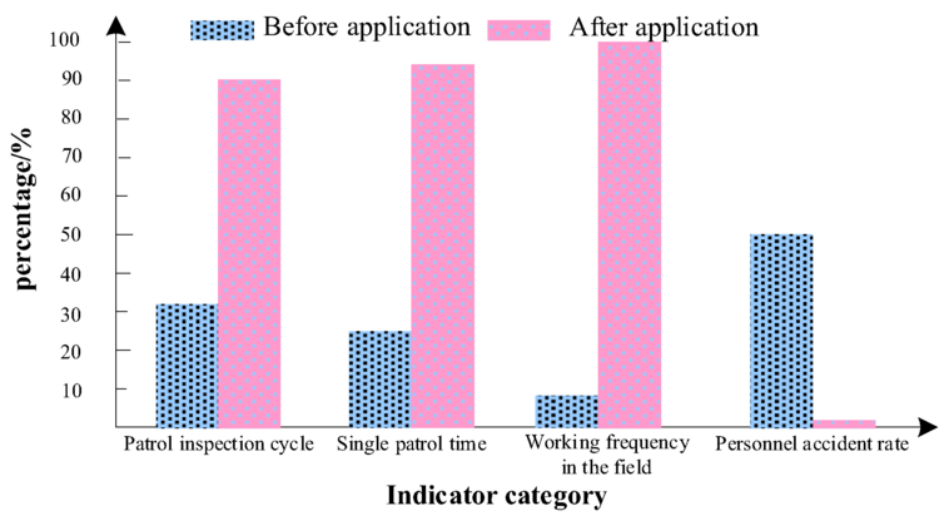

Figure 7. Comparison of practical application effect.

\section{Conclusions}

A real-time early-warning model of transmission tower tilt detection based on multisensor data fusion method is proposed. Through the data collected by sensors, the force distribution of the tower can be analyzed, and the stability of the tower can be judged. At the same time, the tilt angle can be analyzed to judge whether the tower is in a safe state, so as to realize accurate alarm. The effects before and after the application of the model show that the model can effectively ensure the personal safety of staff, improve work efficiency and reduce work intensity. The application of the results of this paper can effectively solve the problem that it is difficult to find the inclination of transmission tower in time by manual monitoring.

\section{References}

[1] Tang Yi, Xu Xiangxiang, Chen Bin, Yi Tao. Space-Time-Intensity online early-warning of transmission tower faults by caused rainfall-induced landslides. Electric Power, 2020, 53(1): 56-65.

[2] Yang Zhi, Ou Wenhao, Fei Xiangze, Li Chaung, Ma Xiao, Zhao Binbin. Smart identification of transmission tower based on high-resolution SAR image and deep learning. Electrical Measurement \& Instrumentation, 2020, 57(4): 71-77.

[3] Chen Wencan, Meng Xiaobo, Zhang Haipeng, Yang Daiming, Mei Hongwei, Wang Liming. Development of on-site real-time monitoring system for corrosion of power transmission tower foots. High Voltage Apparatus, 2020, 56(12): 196. 
[4] Huang Xinbo, Zhao Long, Chen Ziliang, Liu Cheng. An online monitoring technology of tower foundation deformation of transmission lines. Structural health monitoring, 2019, 18(3):949-962.

[5] Hou Hui , Yu Shiwen, Wang Hongbin, Huang Yong, Wu Hao, Xu Yan, Li Xianqiang, Geng Hao. Risk assessment and its visualization of power tower under typhoon disaster based on machine learning algorithms. Energies, 2019, 12(2).

[6] Chen Qiang, Wang Jian, Xiong Xiaofu, Feng Changyou, Ma Chao. Monitoring and early warning method for transmission tower under rainfall-induced landslide disaster. Power System Protection and Control, 2020, 48(3).

[7] An Liqiang, Guan Yongyu, Zhu Zhijian, Zhang Ronglun. Research on windage yaw flashovers of transmission lines under wind and rain conditions. Energies, 2019, 12.

[8] Zhang Anan, Deng Fangming. Low Delay Technology of Transmission Line Tower Monitoring Network Integrating Wireless Sensor Network and Radio Frequency Identification. Science Technology and Engineering, 2020, 20(4).

[9] Du Hao, Huang Huan, Mao Xianyin, Lv Qianyong, Zhou Zhenhua, Huang Li. Distributed monitoring of wind deflection angle of insulator on transmission line. Telecom Power Technology, 2017, 34(6). 